\title{
Knowledge, Attitude, and Practice of Dental Health Professionals Toward Management of Dental Waste in the Aseer Region, Kingdom of Saudi Arabia
}

\author{
Ali A Asiri ${ }^{1}$, Mohammad A Baseer ${ }^{2}$, Jamal A Alsanea ${ }^{3}$, Sultan Binalrimal ${ }^{4}$, Asma H Almeslet ${ }^{5}$, Ghada Alotaibi $^{6}$
}

\begin{abstract}
Introduction: During the process of oral healthcare, infectious and toxic waste is produced within the clinics. Hence, dental professionals are required to know in depth about the proper management of dental waste in order to minimize the risks of cross-infection and the effects of toxins released into the environment affecting human health.

Objectives: To assess the knowledge, attitudes, and practices of dental health professionals (DHP) toward management of dental waste in the Aseer region of Kingdom of Saudi Arabia.

Materials and methods: A descriptive cross-sectional study was carried out among dentist, dental hygienist, dental assistant (DHP) practicing in the Aseer region of Kingdom of Saudi Arabia. A structured, close-ended and self-administered questionnaire consisting of 26 items along with the demographic information was distributed to the $450 \mathrm{DHP}$, and 431 responses were obtained and analyzed for the knowledge, attitude, and practices toward dental waste management.

Results: The mean knowledge, attitude, and practice scores toward dental waste management were found to be $10.95 \pm 1.98,6.50 \pm 1.07$, and $3.07 \pm 1.02$, respectively. Female DHP and those working in the private dental clinics demonstrated a significantly higher knowledge, attitude, and practice scores compared to their counter parts. Knowledge of dental waste management differed significantly in different age groups, while the experience of DHP demonstrated significant differences in knowledge as well as the attitude of DHP toward dental waste management. Dental assistants demonstrated the highest knowledge, attitude, and practice scores of dental waste management compared to the dental hygienist and dentist. DHP showed a significant positive correlation between knowledge-attitude $(r=0.214, p=0.000)$, knowledge-practice $(r=0.208, p=0.000)$, and attitude-practice $(r=0.206, p=0.000)$.

Conclusion: DHP participated in this study demonstrated a high knowledge, positive attitude, and inadequate dental waste management practices in the Aseer region of Kingdom of Saudi Arabia. Hence, there is a need for regular training, workshops, and continuous educational programs on dental waste management.

Keywords: Attitude, Dentist, Dental assistant, Dental hygienist, Dental waste management, Knowledge, Practice.

Journal of Oral Health and Community Dentistry (2019): 10.5005/jp-journals-10062-0048
\end{abstract}

\section{INTRODUCTION}

Dental waste refers to the materials used up in the dental clinics that are no further usable; as a result, they are discarded. In general, medical and dental clinics produce a variety of wastes that are classified into: hazardous and non-hazardous waste, biohazardous waste, as well as sharps and pharmaceutical waste. The waste produced in dental clinics is similar to that generated by other hospitals. This includes a large component of general waste and a smaller proportion of hazardous waste. 'I has been pointed out that dental waste consists of a notable amount of recyclable materials. ${ }^{2}$

Dental professionals are at a great risk for acquiring infection during treatment of patients, as most human pathogens have been isolated from oral secretions. ${ }^{3}$ Within the dental clinical setups, various dental instruments and materials are exposed to the blood and saliva, which act as impending sources of infection. Moreover, chemical substances such as acrylics, impression materials, and mercury used for the restorative purposes could have an adverse health impact if not managed properly.

Different types of organisms present in the infectious waste can gain access into the human body by itching, cutting through the skin and oral mucosa, inhalation, or ingestion. Infectious waste constitutes the vital part of the dental waste made up of contaminated blood and body fluids as well as sharp and
${ }^{1}$ Department of Dental Public Health and Advanced Education General Dentistry, Riyadh Elm University, Riyadh, Kingdom of Saudi Arabia

${ }^{2,3}$ Department of Preventive Dentistry, Riyadh Elm University, Riyadh, Kingdom of Saudi Arabia

${ }^{4,6}$ Department of Restorative Dentistry, Riyadh Elm University, Riyadh, Kingdom of Saudi Arabia

${ }^{5}$ Department of Oral Maxillofacial Surgery and Diagnostic Sciences, Riyadh Elm University, Riyadh, Kingdom of Saudi Arabia

Corresponding Author: Ali A Asiri, Department of Dental Public Health and Advanced Education General Dentistry, Riyadh Elm University, Riyadh, Kingdom of Saudi Arabia, Phone: +966 555758938, e-mail: ali. a1.asiri@student.riyadh.edu.sa

How to cite this article: Asiri AA, Baseer MA, et al. Knowledge, Attitude, and Practice of Dental Health Professionals Toward Management of Dental Waste in the Aseer Region, Kingdom of Saudi Arabia. J Oral Health Comm Dent 2019;13(2):34-38.

Source of support: Nil

Conflict of interest: None

cutting items. Previous studies have reported the possibility of transmission of the serious infections such as AIDS, hepatitis B and $C$ through the dental waste. Improper biomedical waste 
management could lead to a wide spread transfer of pathogens in developing countries. ${ }^{2,4}$

A study on the microbial content of solid waste produced in a dental setting in Brazil identified more than 700 bacterial strains. Staphylococcus, Enterococcus faecalis, Stenotrophomonas were commonly isolated species from the dental solid waste. Clinically important bacterial species with resistance and biofilm profiles could be obtained from the infectious dental waste. There is always a potential risk of transfer of these microorganisms to waste handlers, professionals, and patients in cases of negligence. ${ }^{5}$ Chemical and toxic waste produced within the dental clinics mainly comes from the polluted amalgam, lead foil, disinfectant solutions, metals, and other dental materials utilized in the dentistry. ${ }^{4,6}$

Dental professionals are required to know in depth about the proper management of dental waste in order to minimize the risks of cross infection and effects of toxins released into the environment. To provide guidance to the dental health professionals, Ministry of Health General Directorate for Infection Prevention and Control and Directorate of Dentistry developed an infection prevention and control manual for dental settings. This manual describes the policy and procedures that the dental healthcare personnel are expected to follow in the dental practice. It outlines the primary responsibilities of dental practitioners in relation to infection prevention and control.

The Aseer region is located southwest of the Kingdom of Saudi Arabia, having an area of $76,693 \mathrm{Sq} \mathrm{km}$ and an estimated population of $2,211,875$. The region consists of 13 governorates. Dental services are provided through the network of ministry of health dental clinics, King Khalid University Dental Clinics, and Private Dental Practitioners. One of the studies conducted in the Aseer region has highlighted gaps in solid waste management among the laboratory technicians working in private dental clinics and independent dental laboratories. ${ }^{7}$ In general, reports published in regard to compliance and adherence of dental health professionals toward the dental waste management from Kingdom of Saudi Arabia have indicated variable degrees of awareness and practices. Particularly, there is a lack of in-depth information on knowledge, attitude, and practices of dental professionals from the Aseer region toward dental waste management. Hence the present study aimed to evaluate the knowledge, attitudes, and practices of dental health professionals toward management of dental waste in the Aseer region of Kingdom of Saudi Arabia

\section{Materials and Methods}

\section{Ethical Approval}

The study proposal was submitted to the research center of Riyadh Elm University (Registration Number FPGRP/437310019/230). After careful examination, the institutional review board formally approved the study (IRB number RC/IRB/2018/1124). An informed consent to participate in the study was obtained from the dental professionals.

\section{Study Design and Setting}

This work is a descriptive cross-sectional study involving dental health professionals (dentists, dental hygienists, and assistants) practicing in government and private dental clinics in the Asser region of Kingdom of Saudi Arabia.

\section{Sample}

A list of all the private and government dental clinics was prepared by using the ministry of health portal and relevant websites in 13 governorates of the Aseer region. Then, by employing a convenient sampling methodology, few dental clinics were selected from the governorates, and then DHP working in these clinics were considered into the study.

\section{Sample Size Calculation}

A sample size of 431 DHP was calculated by considering effect size $(f=0.19)$, alfa error probability $(0.05)$, power $(0.95)$, and number of three groups (dentist, dental assistants, and dental hygienists). This sample calculation was performed by using a G Power 3.1.3 sample size calculator.

\section{Measuring Instrument}

After a thorough review of literature and guidelines of biomedical waste management issued from health authorities in Kingdom of Saudi Arabia, a structured, close-ended, and self-administered questionnaire was designed in English language and sent for the expert opinion in REU. Validity and reliability (Cronbach's alpha $=$ 0.80 ) were found to be satisfactory.

\section{Questionnaire Contents}

The questionnaire (Proforma) consisted of four main parts: the first part elicited personal information of the DHP (age, gender, DHP, years of practice, and practice location), the second part had 12 questions on the knowledge of dental waste management, the third part included 8 questions eliciting attitude of DHP toward dental waste management, and the fourth part assessed the practices of DHP toward the dental waste management through 6 items. A total of 450 questionnaire copies were printed and distributed to the DHP. A single investigator visited the various dental clinics in the Aseer region to distribute the questionnaires to the DHPs in their offices during the working hours. Data collection was carried out over a period of three months (October-December 2018). It took 5-7 minutes to complete the questionnaire by the DHP. In total, 450 questionnaires were distributed and 19 were excluded owing to incomplete filling up of information. Therefore, a total of 431 subjects were included, coded, and entered into the excel sheet. The most appropriate response from every questionnaire was scored as 1 and all the others were scored as zero. The knowledge domain score ranged 0-12; attitude score 0-8; and practice scores 0-6.

\section{Statistical Analysis}

Normality was assessed by applying the Shapiro-Wilks test and data were found to be normally distributed $(p<0.05)$. Frequency distribution and percentages were calculated for the characteristics of the study participants. Mean and standard deviation values were calculated for the knowledge, attitude, and practices of DHP toward dental waste management. Non-parametric MannWhitney $U$ tests were applied to compare the knowledge, attitude, and practices between gender and work places of the subjects. While Kruskal-Wallis tests were applied to assess the knowledge, attitude, and practices with regard to the age, years of experience, and category of DHP. Spearman correlation coefficient test was applied to assess the relationship between knowledge, attitude, and practices of dental waste management among DHP. All the analysis was performed by using statistical software package SPSS 
(version 25). A $p$ value $<0.05$ was considered statistically significant in all statistical inferences.

\section{Results}

Of the 450 questionnaires distributed, 431 were considered for the final analysis, thereby making a response rate of 95.77. Table 1 shows the distribution of the personal characteristics of the study participants. Most of the DHP participated in the study were females (229 (53.1\%)) when compared to the males (202 (46.9\%)). Majority of the female dentists, aged $22-32$ years having $1-5$ years of experience

Table 1: Distribution of personal characteristics of the study participants

\begin{tabular}{lllr}
\hline Variables & & $n$ & $\%$ \\
\hline Gender & Male & 202 & 46.9 \\
& Female & 229 & 53.1 \\
& Total & 431 & 100.0 \\
DHP & Dentist & 229 & 53.1 \\
& Dental hygienist & 25 & 5.8 \\
& Dental assistant & 177 & 41.1 \\
& Total & 431 & 100.0 \\
Age (years) & $22-32$ & 229 & 53.1 \\
& $33-42$ & 143 & 33.2 \\
& $\geq 43$ & 59 & 13.7 \\
Experience (years) & Total & 431 & 100.0 \\
& 6-10 & 230 & 53.4 \\
& $\geq 10$ & 103 & 23.9 \\
& Total & 98 & 22.7 \\
Work place & Government & 431 & 100.0 \\
& Private & 244 & 43.4 \\
Age (years) & Total & 431 & 56.6 \\
Experience (years) & Mean \pm SD & $33.72 \pm 7.61$ & 100.0 \\
Knowledge score (0-12) & Mean \pm SD & $7.12 \pm 6.22$ & \\
Attitude score (0-8) & Mean \pm SD & $10.95 \pm 1.98$ \\
\hline
\end{tabular}

and working mainly in private dental clinics participated in the study. The mean age and years of experience of the study participants were $33.72 \pm 7.61$ years and $7.12 \pm 6.22$ years, respectively. Mean knowledge, attitude, and practice scores toward dental waste management were found to be $10.95 \pm 1.98,6.50 \pm 1.07$, and $3.07 \pm 1.02$, respectively.

Female DHPs demonstrated a significantly higher knowledge $(11.23 \pm 1.63$ vs $10.64 \pm 2.28, p=0.005)$, attitude $(6.63 \pm 0.92$ vs $6.36 \pm 1.20, p=0.036)$, and practice $(3.18 \pm 1.02$ vs $2.94 \pm 1.01)$ scores toward dental waste management than the males. Similarly, DHP working in private dental clinics showed asignificantly higher mean knowledge (11.34 \pm 1.32 vs $10.44 \pm 2.52, p=0.009)$, attitude $(6.71 \pm 0.85$ vs $6.23 \pm 1.24, p=0.000)$ and practices $(3.29 \pm 1.04$ vs $2.78 \pm 0.93, p=0.000)$ scores toward dental waste management compared to the government DHPs (Table 2).

When mean knowledge scores of dental waste management was compared across different age groups (22-32 years, $33-42$ years and $\geq 43$ years), a statistically significant difference was observed $(10.60 \pm 2.30,11.28 \pm 1.60,11.54 \pm 0.97, p=0.000)$ The mean knowledge score was significantly lower in those aged $22-32$ years compared to those aged $33-42$ years and $\geq 43$-year-old DHP. However, attitude $(p=0.170)$ and practice $(p=680)$ scores did not show any significant differences across different age groups. Similarly, the knowledge ( $1-5,6-10$ and $\geq 10$ years) and attitude $(1-5,6-10$ and $\geq 10$ years) of DHP showed statistically significant difference $(10.71 \pm 2.14,11.05 \pm 2.17,11.43 \pm 1.13, p=0.007)$ and $(6.35 \pm 1.20,6.57 \pm 0.88,6.79 \pm 0.83, p=0.010)$ with regard to their years of experience. DHP with 1-5 years of experience showed significantly lower knowledge and attitude scores compared to those with $\geq 10$ years of experience (Table 3 ).

Dentist, dental hygienist, and dental assistants demonstrated mean knowledge $(10.60,11.48$, and 11.32$)$, attitude $(6.47,6.48$, and 6.55), and practice (3, 2.88 and 3.19) scores. Dental hygienist demonstrated a significantly higher knowledge compared to dentists toward dental waste management. However, attitude and practice scores did not differ significantly among DHP (Fig. 1).

DHP showed significant positive correlation between knowledge-attitude $(r=0.214, p=0.000)$, knowledge-practice $(r=0.208, p=0.000)$, and attitude-practice $(r=0.206, p=0.000)$ as shown in (Table 4).

Table 2: A comparison of knowledge, attitude, and practice scores between gender and workplace

\begin{tabular}{|c|c|c|c|c|c|c|c|c|c|c|}
\hline \multirow[b]{2}{*}{ Scores } & \multicolumn{2}{|c|}{ Male } & \multicolumn{2}{|c|}{ Female } & \multirow[b]{2}{*}{$p^{*}$} & \multicolumn{2}{|c|}{ Government } & \multicolumn{2}{|c|}{ Private } & \multirow[b]{2}{*}{$p^{*}$} \\
\hline & Mean & $S D$ & Mean & $S D$ & & Mean & $S D$ & Mean & $S D$ & \\
\hline Knowledge & 10.64 & 2.28 & 11.23 & 1.63 & 0.005 & 10.44 & 2.52 & 11.34 & 1.32 & 0.009 \\
\hline Attitude & 6.36 & 1.20 & 6.63 & 0.92 & 0.036 & 6.23 & 1.24 & 6.71 & 0.85 & 0.000 \\
\hline Practice & 2.94 & 1.01 & 3.18 & 1.02 & 0.012 & 2.78 & 0.93 & 3.29 & 1.04 & 0.000 \\
\hline
\end{tabular}

*Mann-Whitney $U$ test; SD, standard deviation

Table 3: A comparison of knowledge, attitude, and practice scores across age and experience groups

\begin{tabular}{|c|c|c|c|c|c|c|c|c|c|c|c|c|c|c|}
\hline \multirow[b]{3}{*}{ Scores } & \multicolumn{6}{|c|}{ Age (years) } & \multirow[b]{3}{*}{$p^{\S}$} & \multicolumn{6}{|c|}{ Experience (years) } & \multirow[b]{3}{*}{$p^{\S}$} \\
\hline & \multicolumn{2}{|c|}{$22-32$} & \multicolumn{2}{|c|}{$33-42$} & \multicolumn{2}{|c|}{$\geq 43$} & & \multicolumn{2}{|c|}{$1-5$} & \multicolumn{2}{|c|}{$6-10$} & \multicolumn{2}{|c|}{$\geq 10$} & \\
\hline & Mean & $S D$ & Mean & $S D$ & Mean & $S D$ & & Mean & $S D$ & Mean & $S D$ & Mean & $S D$ & \\
\hline Knowledge & 10.60 & $2.30^{\mathrm{a}}$ & 11.28 & $1.60^{\mathrm{b}}$ & 11.54 & $0.97^{b}$ & 0.000 & 10.71 & $2.14^{a}$ & 11.05 & 2.17 & 11.43 & $1.13^{b}$ & 0.007 \\
\hline Attitude & 6.41 & 1.16 & 6.57 & 0.93 & 6.71 & 0.95 & 0.170 & 6.35 & $1.20^{\mathrm{a}}$ & 6.57 & 0.88 & 6.79 & $0.83^{b}$ & 0.010 \\
\hline Practice & 3.04 & 1.11 & 3.07 & 0.96 & 3.17 & 0.81 & 0.680 & 3.04 & 1.10 & 3.18 & 1.00 & 3.01 & 0.84 & 0.408 \\
\hline
\end{tabular}

${ }^{8}$ Kruskal-Wallis test, different letters across the rows indicate significant differences between groups 


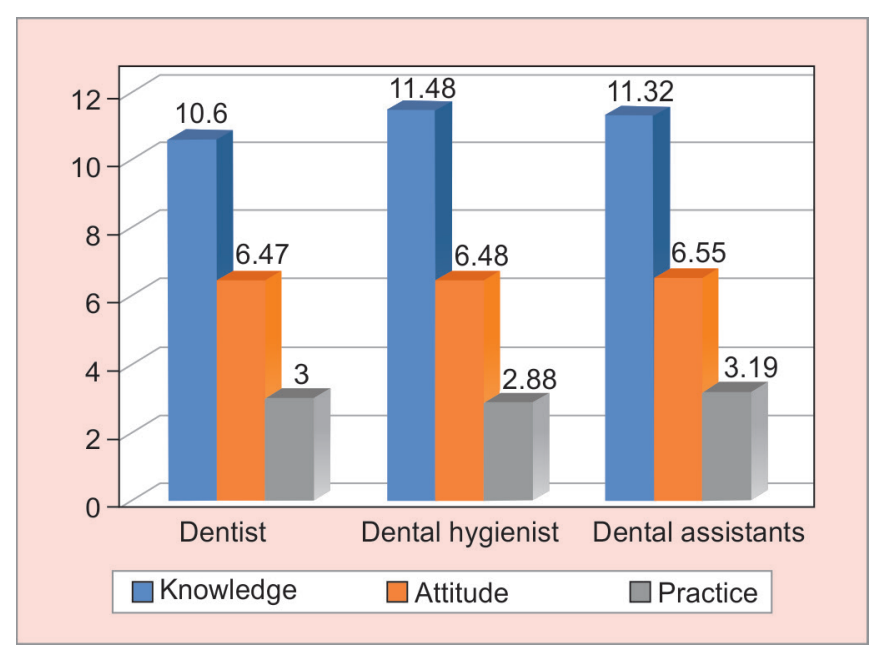

Fig. 1: A comparison of mean knowledge, attitude, and practice scores among DHP

Table 4: Spearman correlation between knowledge, attitude, and practices

\begin{tabular}{lllll}
\hline Variables & & Knowledge & Attitude & Practice \\
\hline Knowledge & $\begin{array}{l}\text { Correlation } \\
\text { coefficient }\end{array}$ & 1.000 & $0.214^{* *}$ & $0.208^{* *}$ \\
& Sig. (2-tailed) & - & 0.000 & 0.000 \\
& $N$ & 431 & 431 & 431 \\
Attitude & Correlation & $0.214^{* *}$ & 1.000 & $0.206^{* *}$ \\
& coefficient & & & \\
& Sig. (2-tailed) & 0.000 & - & 0.000 \\
& $N$ & 431 & 431 & 431 \\
Practice & Correlation & $0.208^{* *}$ & $0.206^{* *}$ & 1.000 \\
& coefficient & & & \\
& Sig. (2-tailed) & 0.000 & 0.000 & - \\
& $N$ & 431 & 431 & 431 \\
\hline
\end{tabular}

**Correlation is significant at the 0.01 level (2-tailed)

\section{Discussion}

Dental waste produced within the clinical setting is made up of infectious, chemical and hazardous materials. This waste management is a complicated issue if not handled properly, as it exposes healthcare professionals, healthcare facilities, waste handlers and the community at risk of infectious diseases, toxicity, and injury. Hence healthcare facilities have a primary duty of care for the surrounding environment and public health. It is the responsibility of the facilities to take care of the waste produced by them. ${ }^{8}$ Inadequate sterilization and waste management in dental clinics were considered as the top one ethical challenge. ${ }^{9}$

Knowledge is an essential measure that permits to discriminate between right and the wrong; it is a combination of comprehension, experience, judgment, and skill. Attitude recognizes thinking toward a proper situation. Practice refers to the action based on contemplation of rules and the knowledge. Hence, a correct knowledge of the subjects with a positive attitude and a good practice are essential to guide and serve the patients. ${ }^{10,11}$ Assessment of knowledge, attitude, and practices of DHP is an important aspect of improving dental waste management strategies. Hence the present study explored the DHP knowledge, attitude, and practices toward dental waste management in the Aseer region of Kingdom of Saudi Arabia.

In general, DHP showed a very high knowledge (10.95 \pm 1.98 , range $0-12$ ) toward the dental waste management. This finding is contrary to the studies reported various studies in which a lower level of knowledge observed among the DHP and dental students. ${ }^{11-13}$ However, other studies have reported adequate knowledge of policies on biomedical waste management. ${ }^{14,15}$ The reason for such increased knowledge of dental waste management may be related to the regulatory authorities ( $\mathrm{MOH}$ and environment) that makes it compulsory for the medical care and dental care facilities to adhere to the standards of biomedical waste management. Moreover, participation of the DHW in seminars and workshops emphasize waste management methods. A high percentage (97\%) of DHP acknowledged the responsibility to follow and respect all aspects of infection prevention and control policy. Similarly, a high percentage (97\%) of DHP responded positively to the fact that the dental waste can be potentially infectious and can transmit diseases such as HIV/ hepatitis. In contrast, a relatively low (81\%) response was observed with non-regulated general medical waste produced within the dental clinics, that can be disposed of with the general office trash.

Female DHP showed a high level of knowledge toward dental waste management. One of the reasons for this could be a higher participation of females in the survey than males.

DHP working in the private sector demonstrated a significantly higher knowledge score compared to the government sector $(p=0.000)$. Private dental clinics license renewal requires a periodic inspection of dental waste management compliance by relevant authorities. Hence, private dental clinics need to be particular about the dental waste management. Hence, DHP working in the private sector should be equipped with required knowledge of dental waste management. ${ }^{16}$

Knowledge of dental waste management improved significantly as the age $(p=0.000)$ and experience $(p=0.007)$ of the DHP increased, suggesting a repeated exposure to information related to the dental waste management. Interestingly, dental assistants and dental hygienist showed a higher knowledge of dental waste management compared to the dentists. This finding is in line with that reported by Singh et al., who demonstrated that the paramedical staff had a good theoretical knowledge of dental waste management. ${ }^{3}$ However, other study reported variable and inconsistent knowledge of waste management dental practitioners. $^{17}$

Female DHP and those working in government sector demonstrated a significantly higher attitude and practice scores, suggesting high compliance toward the dental waste management. However, increase in the age did not show any significant effect on attitude and practices although there is a significant difference in mean knowledge score toward dental waste management. As the experience increased $\geq 10$ years, the attitude toward dental waste management likely to increase without having any significant influence on practice of dental waste management. This finding suggests that years of experience is more important to influence the attitude of DHP of dental waste management rather than the age.

Dental assistants showed higher attitude and practice scores compared to the dental hygienist and dentist. This could be due to their high knowledge and participation in waste management training programs. In general, correlation statistics pointed out that DHP with adequate knowledge demonstrated positive attitude and practices toward dental waste management. Biomedical waste 
management rules mandate that healthcare personnel should have a proper knowledge and practice of dealing with and disposal of biomedical waste. ${ }^{18}$

The precision of a self-administered questionnaire depends mainly on the way questions have been written, its analysis, and response rate. In order to receive a good response rate and accuracy of the questionnaire items, close-ended, self-administered questionnaires were utilized. The study findings are applicable only to the DHP working in the Aseer region. This study results are mainly based on self-reports of the DHP, which might have led to the overestimation of compliance.

Hence there is a need to conduct further studies in all the 13 regions of the Kingdom of Saudi Arabia to know the existing knowledge, attitude, and practices of dental waste management among DHP. Another limitation of the study is that the amount and the type of dental waste produced from various dental clinics have not been measured.

\section{Conclusion}

DHP participated in this study demonstrated a high knowledge, positive attitude, and inadequate dental waste management practices in the Aseer region of Kingdom of Saudi Arabia. Hence, there is a need for regular training, workshop, and continuous educational programs on dental waste management among different categories of DHP.

\section{References}

1. Sudhakar V, Chandrashekar J. Dental health care waste disposal among private dental practices in Bangalore City, India. Int Dent J 2008 Feb;58(1):51-54. DOI: 10.1111/j.1875-595X.2008.tb00176.x.

2. Momeni H, Tabatabaei Fard SF, et al. Composition, Production Rate and Management of Dental Solid Waste in 2017 in Birjand, Iran. Int J Occup Environ Med 2018;9(1):52-60. DOI: 10.15171/ijoem.2018. 1203.

3. Singh BP, Khan SA, et al. Current biomedical waste management practices and cross-infection control procedures of dentists in India. Int Dent J 2012 Jun;62(3):111-116. DOI: 10.1111/j.1875595X.2011.00100.x.

4. Ghanbarian M, Khosravi A, et al. Evaluation of Quantity and Quality of Dental Solid Waste. Knowl Health 2011;6:43-46.

5. Tagliaferri TL, Vieira CD, et al. Phenotypic and genotypic characterization of clinically relevant bacteria isolated from dental waste and waste workers' hands, mucosas and coats. Lett Appl Microbiol 2017 Oct;65(4):306-312. DOI: 10.1111/lam.12775.
6. Vieira CD, de Carvalho MAR, et al. Count, identification and antimicrobial susceptibility of bacteria recovered from dental solid waste in Brazil. Waste Manag 2011 Jun;31(6):1327-1332. DOI: 10.1016/ j.wasman.2010.12.016.

7. Haralur SB, Al-Qahtani AS. The Dental Solid Waste Management in Different Categories of Dental Laboratories in Abha City, Saudi Arabia. Open Dent J 2015;9:449-454. DOI: 10.2174/1874210601509010449.

8. Basu M, Das P, et al. Assessment of future physicians on biomedical waste management in a tertiary care hospital of West Bengal. J Nat Sci Biol Med 2012;3(1):38-42. DOI: 10.4103/0976-9668.95945.

9. Kemparaj VM, Panchmal GS, et al. The Top 10 Ethical Challenges in Dental Practice in Indian Scenario: Dentist Perspective. Contemp Clin Dent 2018;9(1):97-104. DOI: 10.4103/ccd.ccd_802_17.

10. Jain M, Sawla L, et al. Knowledge, attitude and practice towards droplet and airborne isolation precautions amongs dental health care professionals in India. Med Oral Patol Oral Cirugia Bucal 2010; 15(6):e957-e961. DOI: 10.4317/medoral.15.e957.

11. Sanjeev R, Kuruvilla S, et al. Knowledge, attitude, and practices about biomedical waste management among dental healthcare personnel in dental colleges in Kothamangalam: a cross-sectional study. Health Sci 2014;1(3):1-12.

12. Singh T, Ghimire TR, et al. Awareness of Biomedical Waste Management in Dental Students in Different Dental Colleges in Nepal. BioMed Res Int 2018;2018:1742326. DOI: 10.1155/2018/ 1742326.

13. Narang RS, Manchanda A, et al. Awareness of biomedical waste management among dental professionals and auxiliary staff in Amritsar, India. Oral Health Dent Manag 2012;11(4):162-168.

14. Raghuvanshi M, SinhaS. Awareness of Biomedical Waste Management among Dentists associated with Institutions and Private Practitioners of North India: a Comparative Study. J Contemp Dent Pract 2018; 19(3):273-277. DOI: 10.5005/jp-journals-10024-2251.

15. Mugabi B, Hattingh S. Assessing knowledge, attitudes, and practices of healthcare workers regarding medical waste management at a tertiary hospital in Botswana: a cross-sectional quantitative study. Niger J Clin Pract 2018;21(12):1627. DOI: 10.4103/njcp. njcp_270_17.

16. Al-Rabeah A, Moamed AG. Infection control in the private dental sector in Riyadh. Ann Saudi Med 2002;22(1-2):13-17. DOI: 10.5144/0256-4947.2002.13.

17. Chaudhari K, Patel J, et al. Knowledge, attitude and practices among dentists regarding bio-medical waste management in Ahmedabad City, Gujarat: a questionnaire-based study. J Oral Health Med Res 2015;2:23-26.

18. Ismail M, Kulkarni AG. Knowledge, attitude and practice about biomedical waste management among personnel of a tertiary health care institute in Dakshina Kannada, Karnataka. Al Ameen J Med Sci 2013;6:376-380. 